\title{
Computer-generated facial areas of interest in eye tracking research: A simulation study
}

${ }^{1}$ Biological and Clinical Psychology, Department of Psychology, University of Trier, Trier,

* Correspondence:

6 Gregor Domes

7 domes@uni-trier.de 


\section{FACIAL AREAS OF INTEREST IN EYE TRACKING}

Abstract

Advances in eye tracking technology have enabled the development of interactive experimental setups to study social attention. Since these setups differ substantially from the eye

24 tracker manufacturer's test conditions, validation is essential with regard to data quality and other

25 factors potentially threatening data validity.

In this study, we evaluated the impact of data accuracy and areas of interest (AOIs) size on

27 the classification of simulated gaze data. We defined AOIs of different sizes using the Limited-

28 Radius Voronoi-Tessellation (LRVT) method, and simulated gaze data for facial target points with

29 varying data accuracy. As hypothesized, we found that data accuracy and AOI size had strong effects

30 on gaze classification. In addition, these effects were not independent and differed for falsely

31 classified gaze inside AOIs (Type I errors) and falsely classified gaze outside the predefined AOIs

32 (Type II errors). The results indicate that smaller AOIs generally minimize false classifications as

33 long as data accuracy is good enough. For studies with lower data accuracy, Type II errors can still

34 be compensated to some extent by using larger AOIs, but at the cost of an increased probability of

35 Type I errors. Proper estimation of data accuracy is therefore essential for making informed decisions 36 regarding the size of AOIs.

37 Keywords: eye tracking, areas of interest, accuracy, validity, interactive.

40 Number of words: 3546

41 Number of figures: 4 


\section{FACIAL AREAS OF INTEREST IN EYE TRACKING}

\section{Introduction}

44 Eye tracking, especially in its video-based form, has become a standard method for

45 investigating visual attention in many research areas, including social neuroscience $(1,2)$,

46 psychopharmacology (3-5) and virtual reality (6,7). In recent years, several studies have questioned

47 common reporting standards in this field, or the lack of data quality evaluations for the specific setup

48 in use $(8,9)$, e.g., some studies only report the overall accuracy of hardware determined by the

49 manufacturer under ideal test conditions. The issue of data quality and inadequate reporting standards

50 seems to be increasingly relevant as the field advances to develop more naturalistic, interactive or

51 face-to-face eye tracking applications (10-12). Not only do these setups deviate further in design

52 from the manufacturer's test conditions, but when used in naturalistic interactions, other factors can

53 affect the accuracy of the eye tracking data, such as movements accompanying facial expressions or

54 speech.

AOI-based gaze classification is a popular approach for analyzing eye tracking data $(13,14)$.

This approach determines whether a gaze point or a fixation is directed at a predefined region around

57 a target point, e.g., in face perception, the target would be another person's face, while potential AOIs

would be the eye region or the mouth. Researchers have taken this approach to study, for example,

59 gaze behavior of participants with (sub-)clinical social anxiety or autism $(11,15,16)$. For these

60 applications in potentially interactive scenarios, automatic procedures to generate AOIs seem

61 beneficial (13), as manual generation can be time-consuming depending on the recording duration. In

62 addition, the uniformity of target stimuli in these setups allow the use of standardized, published

63 procedures (e.g. Limited-Radius Voronoi-Tessellation method, LRVT; (17)), thus ensuring the

64 comparability of research results. In this method, AOI size can be easily regulated by adjusting the

65 limiting radius. 
The impact of AOI size on gaze classification has been investigated $(13,14)$. In those studies,

67 suggestions were made that AOIs on sparse stimuli should be large enough to be robust to noise (13),

68 and that oversized AOIs are problematic when considering falsely positive classified gaze data (14).

69 While the problem of inadequate AOI sizes seems to be known, guidelines on how to choose the

70 most appropriate size of AOIs remain vague. In addition, it is conceivable that the choice of AOI

71 sizes depends on the accuracy of eye tracking data. In general, we can assume that low accuracy

72 would require larger AOIs to ensure valid classified gaze points. However, it is unclear how data

73 accuracy and AOI size interact to affect classification performance, and whether false-negatives and

74 false-positives are differentially affected.

With the current study, we aimed to investigate the gaze classification performance as a

76 function of data accuracy and AOI size with simulated gaze data in order to derive guidelines for

77 selecting AOIs and sizing them in interactive (face-to-face) eye tracking applications. Thereby, we

78 focus on classification performance with respect to false-positives (falsely classified inside a specific

79 AOI; Type I error) and false-negatives (falsely classified outside a specific AOI; Type II error) to

80 derive recommendations for choosing AOI size depending on data quality. Specifically, we expected

81 gaze data accuracy and AOI size to independently influence classification performance and for the

82 two factors to interact such that AOI size has a greater impact on classification performance when

83 data accuracy is low. Along with these recommendations, we present a software tool that allows gaze

84 data to be generated with a given accuracy, the visualization of gaze data on AOIs of different sizes,

85 and the evaluation of the resulting classification of gaze data. 


\section{FACIAL AREAS OF INTEREST IN EYE TRACKING}

\subsection{Stimuli}

Facial stimuli from the Face Research Lab London (version 3; DeBruine and Jones, 2017) served as the basis for this simulation. The following four stimuli were selected to represent different

93 ethnic groups: 005 (male, Asian, 28 years old), 012 (male, Caucasian, 24 years old), 025 (female,

94 African American, 21 years old) and 134 (female, Caucasian, 21 years old). A picture with a neutral

95 facial expression and direct gaze was chosen for each stimulus. Stimuli were resized and rescaled to

$96480 \times 480$ px resembling the size of a real person sitting at a viewing distance of approx. $130 \mathrm{~cm}$

97 recorded at $1920 \times 1080 \mathrm{px}$ resolution. After rescaling, the facial stimuli covered an average area of

982.8 by $1.9^{\circ}$, which corresponds to the size of a real face in a face-to-face conversation at the

99 aforementioned viewing distance.

\subsection{Gaze data simulation}

101 The goal for our data simulation was to mimic a standard test procedure with multiple

102 participants and several runs of a gaze validation on facial features, i.e. the instructed sequential

103 fixation of specific targets points on a facial stimulus. For each target, fixations lasting one second

104 each were simulated at a recording frequency of $120 \mathrm{~Hz}$. The coordinates of five facial features (left

105 eye, right eye, nose, mouth \& forehead) were chosen as targets. Each target point corresponded to an

106 AOI center point, except for the forehead point, no AOI was generated. Both target points and AOI

107 center points were determined using OpenFace landmarks (see Fig 1B; (19)).

108 To simulate a realistic set of gaze data for a group of (simulated) participants, the fixation

109 points around the facial targets were determined in several steps. First, a fixed number of virtual

110 participants was generated: Each participant was assigned a random offset angle and a base accuracy

111 derived from a generalized gamma distribution around the intended mean accuracy. The standard 


\section{FACIAL AREAS OF INTEREST IN EYE TRACKING}

112 deviation was set to 0.5 times the mean accuracy and the skewness to 0.6. Second, for each simulated

113 participant, a fixed number of offsets per target was created by varying the individual base accuracy

114 according to normal distribution with a standard deviation of 0.15 times the base accuracy,

115 recalculating all outliers that fell outside three standard deviations. This procedure allowed us to

116 account for within- and between-subjects variance. We applied the above-mentioned method to

117 simulate data with mean accuracy values of $0.5^{\circ}, 1.0^{\circ}$ and $1.5^{\circ}$. The distribution of the simulated data

118 for the three accuracy levels can be found in Fig 1 A.

119 A total of 100 virtual participants were simulated with 30 runs of face validation for each mean

120 accuracy value and stimulus, resulting in 36000 data sets. Data was simulated with an in-house tool

121 written in Python 3.7. The tool can be downloaded here:

122 https://osf.io/ytrh8/?view_only=bedda3995dba447bb58f81c2f8c27880.

\section{$123 \quad 2.3 \quad$ Definition of AOIs}

124 We used the LRVT method (13) with varying radii for facial features and a face ellipse to

125 automatically define AOIs. In the first step, facial landmarks (eyes, nose \& mouth) were obtained

126 using OpenFace (19) - Fig 1B. Second, AOI centers were either derived directly from the facial

127 landmarks (nose \& mouth) or by calculating the midpoint between two landmarks (left \& right eye).

128 The center of the face ellipse is created by calculating the midpoint between the left and right eye

129 corners (x-coordinate) and 1.5 times the distance between facial landmark 8 and 33 (y-coordinate) -

130 Fig 1B. In the final step, AOIs were defined by applying the LRVT method with three different radii

$131\left(1.0,1.5 \& 2.0^{\circ}\right)$ for the facial features and OpenFace landmarks were used to define the face ellipse.

132 The horizontal radius of the ellipse is the smaller distance between the face's center point and facial

133 landmark 0 or 16 (x-coordinate). The vertical radius is the distance between the face center and facial

134 landmark 8 (y-coordinate). To assess the effect of data quality and AOI size on gaze classification 
135 performance, the AOI radius of $4^{\circ}$ proposed in the literature (17) was adjusted to a $131 \mathrm{~cm}$ viewing

136 distance, resulting in a radius of approx. $2.0^{\circ}(\sim 4.6 \mathrm{~cm})$. Additionally, the radius was further reduced

137 twice $\left(1.0^{\circ}[\sim 3.4 \mathrm{~cm}] \& 1.5^{\circ}[\sim 2.3 \mathrm{~cm}]\right)$ to test the effect of different AOI sizes on classification

138 performance.

a) Data simulation

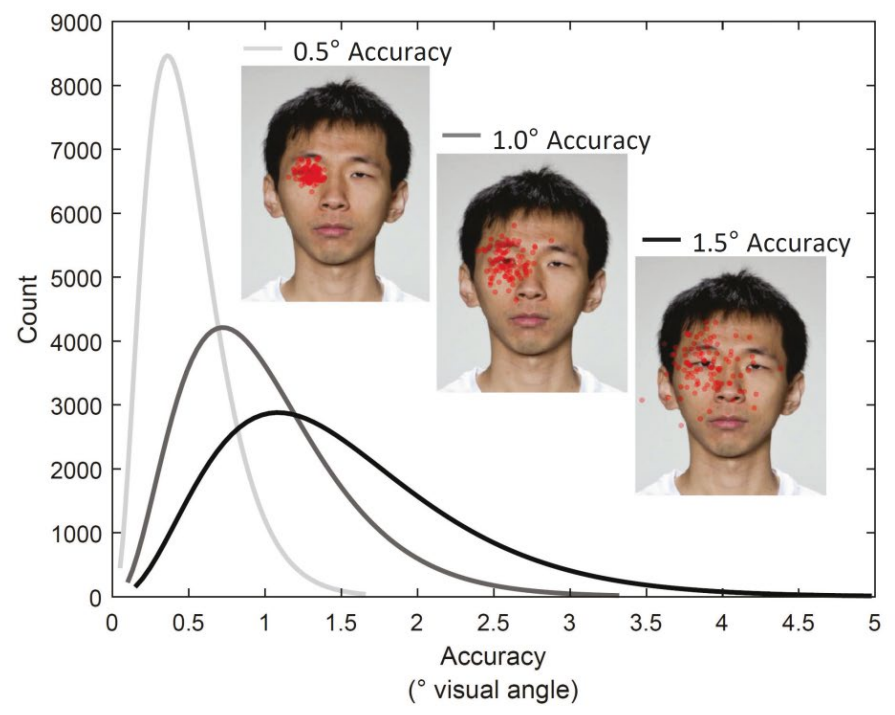

b) Automatic $\mathrm{AOI}$ construction process

1. Facial Landmarks (OF)

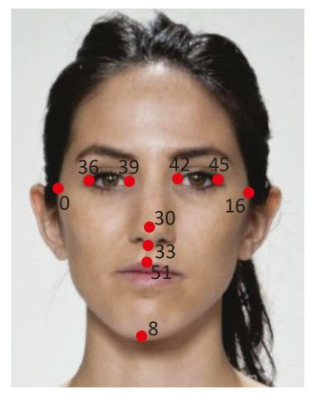

2. AOls centers

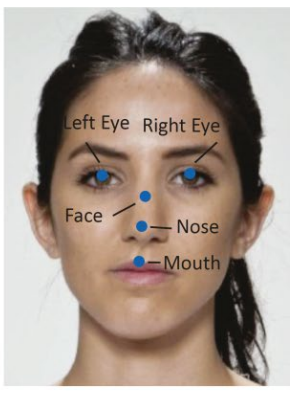

3. Resulting AOls

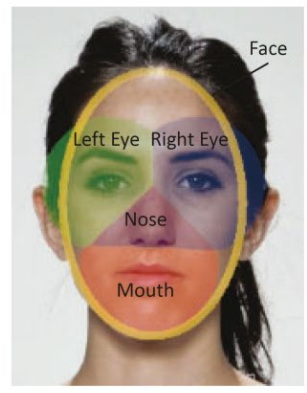

140 Fig 1. Visualization of gaze data simulation and areas of interest (AOI) definition.

141 (A) Visualization of the three gamma functions used to generate gaze data with three levels of

142 accuracy $\left(0.5^{\circ}, 1.0^{\circ} \& 1.5^{\circ}\right)$ and examples of the simulated data for the left eye of a facial stimulus as

143 the fixation target $(n=100$ data sets, averaged over 30 simulated fixations each). (B) Visualization

144 of the three steps of the automatic AOI construction process. 1. Facial landmarks from OpenFace. 2. 
FACIAL AREAS OF INTEREST IN EYE TRACKING

145 AOI center points derived from the facial landmarks. 3. Resulting AOIs using the Limited-Radius

146 Voronoi-Tessellation (LRVT) method (example with 2.0 radius). Note OF = OpenFace (19).

\section{$147 \quad 2.4 \quad$ Data analyses}

148 As a prerequisite for aggregating the data across stimuli, we performed a two-way analysis of

149 variance (ANOVA) with the between-subject factors stimulus (005, 012, 025 \& 134) and accuracy

$150\left(0.5^{\circ}, 1.0^{\circ} \& 1.5^{\circ}\right)$, to test whether the percentage of correctly classified fixation points differed as a

151 function of facial stimulus used for the simulation.

To investigate the influence of data quality and AOI size on false-negatives (Type II error), we analyzed the number of fixation points directed to one of the four facial AOIs that were misclassified

154 as belonging to a different AOI, or to no AOI at all (rest of face \& surrounding). We chose to

155 visualize the effect using confusion matrices and bar plots, and analyzed the data descriptively.

The effect of data quality and AOI size on false-positives (Type I error) was tested by simulating fixation points on the forehead of the facial stimuli for which no AOI had been defined.

158 Classification was correct when no AOI was detected, whereas false-positives occurred when

159 fixations points were misclassified as belonging to one of the AOIs. Again, bar plots were created to

160 visualize the effect of the independent variables, and analyses were performed at the descriptive 161 level. 


\section{FACIAL AREAS OF INTEREST IN EYE TRACKING}

\section{Results}

168 The two-way ANOVA for the percentage of correctly classified fixation points revealed a non169 significant main effect of facial stimulus, $F(3,1188)=1.62, p=.182, \eta^{2}{ }_{\mathrm{G}}<.01$, a significant main

170 effect of condition, $F(2,1188)=408.01, p<.001, \eta^{2} \mathrm{G}=.41$ and a non-significant interaction effect,

$171 F(6,1188)=0.55, p=.768, \eta^{2}{ }_{\mathrm{G}}<.01$, resulting in the aggregation of classification data across 172 stimuli.

\section{$173 \quad 3.1 \quad$ Classification of fixations on facial feature AOIs}

174 Fixations simulated on eyes, nose, and mouth with high data quality, e.g., accuracy values of

$1750.5^{\circ}$, were correctly classified in 82.3 to 97.2 percent of cases. Misclassification and non-

176 classification of fixations (false-negatives; Type II error), in turn, occurred in only 0 to 10.8 percent

177 of cases. In this condition, the classification performance was largely independent of AOI size (Fig 2

178 right column along the vertical axis). With reduced data quality, we noted that differences emerged in

179 the classification performance as a function of AOI size (Fig 2 left and middle column along the

180 vertical axis). Fixations with an accuracy of $1.5^{\circ}$ were on average correctly classified in 22.0 to 60.7

181 percent of the cases. Large AOIs with a radius of $2.0^{\circ}$ resulted in correct classification above chance

182 level for most AOIs (left eye, right eye \& mouth), whereas classification of fixation points on the

183 nose AOI were evenly distributed across all other AOIs, resulting in the highest percentages of false-

184 negatives (19.2 to $27.8 \%$ ). Reducing AOI size, on the other hand, is associated with fewer

185 misclassified fixation points, at the expense of an increase in false-negatives in terms of non-

186 classification (rest of face \& surrounding: 4.3 to $43.7 \%$ ). 


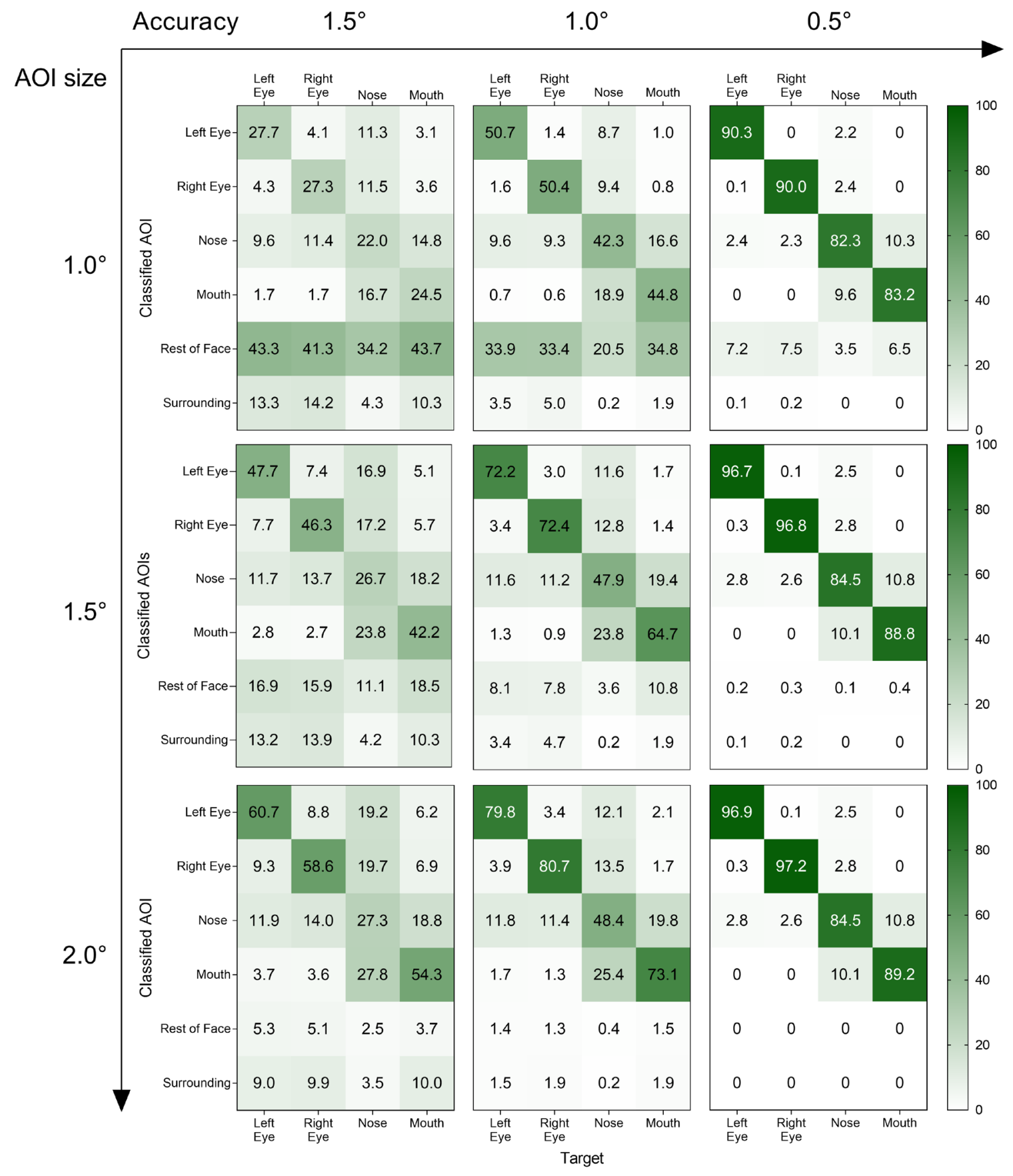

188 Fig 2. Confusion matrices of the fixation classification performance as a function of gaze data

189 accuracy and AOI size.

190 The percentages in the diagonal represent correctly classified fixation points, while the percentages

191 outside the diagonal represent misclassified fixation points. The percentages in the last two rows

192 correspond to the number of unclassified fixation points (rest of face \& surrounding). 


\section{FACIAL AREAS OF INTEREST IN EYE TRACKING}

In a second step we averaged classification performance over all facial target points (see Fig

194 3B). Concerning false-negative classifications, the AOI size seems irrelevant for accuracy values

195 below or equal to $0.5^{\circ}$. For scenarios with accuracy values over $1.0^{\circ}$, larger AOIs result in more

196 correctly classified fixation points at the expense of a slightly increased percentage of misclassified

197 fixation points. Smaller AOIs, on the other hand, lead to fewer misclassified fixation points, but also

198 to a reduction of correctly classified fixation points due to up to $50 \%$ unclassified data-quality-

199 dependent fixation points.

\subsection{Classification of fixations outside AOIs}

To investigate the effect of data quality and AOI size on false-positives (Type I error), data

202 were simulated on a target point for which no AOI had been defined. In this particular case, we

203 simulated data on the forehead of the facial stimuli to recreate a situation in which someone is trying

204 to avoid eye contact and hides their behavior by fixating on the forehead. Fig 3D shows that large

205 AOIs in combination with good data quality $\left(0.5^{\circ}\right.$ accuracy $)$ result in over $90 \%$ misclassified fixation

206 points (inflation of Type I error). As expected, most fixation points were falsely classified as

207 belonging to the left or right eye. Smaller AOIs in combination with good data quality, on the other

208 hand, lower numbers of false-positives drastically with over $80 \%$ of fixation points being correctly

209 classified outside any specific AOI. The effect of data quality is inverse, while smaller AOIs profit

210 from better accuracy in terms of Type I error, the error increases with better accuracy for larger

211 AOIs. 
Classification for gaze inside AOls

a)

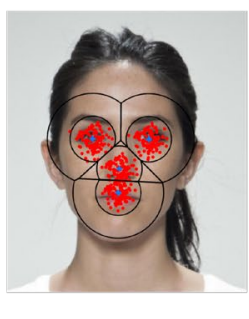

b)

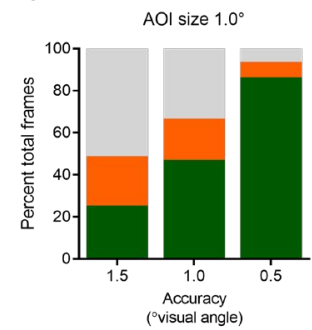

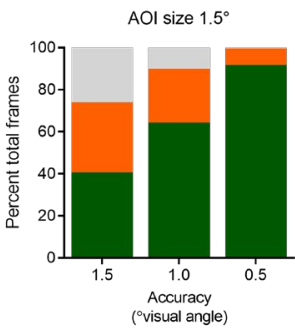
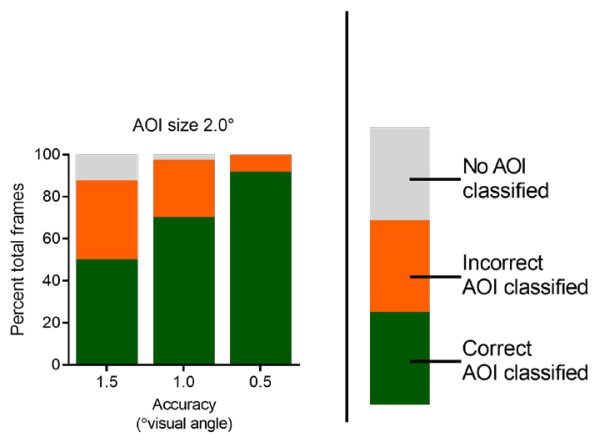

Classification for gaze on forehead

c)

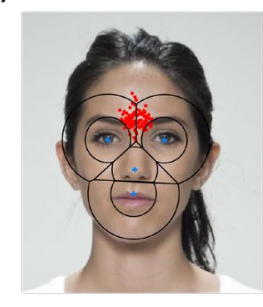

d)

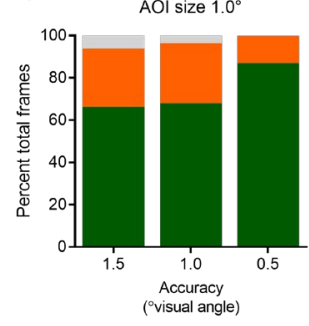

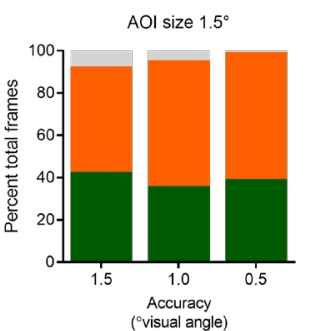

("visual angle)
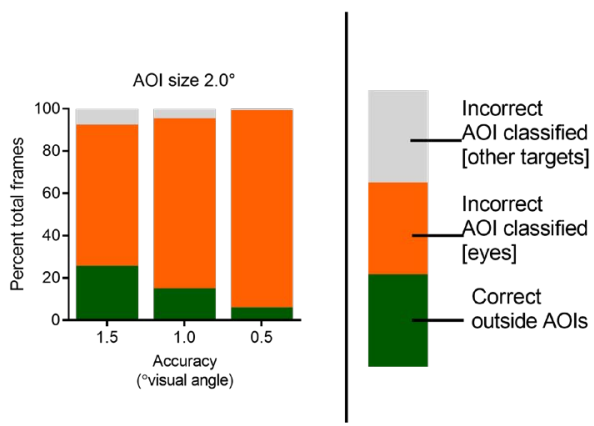

213 Fig 3. Effect of gaze data accuracy and AOI size on classification performance of simulated

214 fixations (on eyes, nose \& mouth) averaged over all facial AOIs.

215 (A) Visualization of the AOI sizes $1.0^{\circ}$ and $2.0^{\circ}$ drawn around the blue center points and the

216 simulated fixation points in red $\left(n=100\right.$, averaged over 30 simulated fixations; accuracy $\left.0.5^{\circ}\right)$.

217 Green indicates correct classification within the corresponding AOI, orange and gray indicate

218 misclassifications as fixations within the other AOIs, or no AOI at all. Effect of gaze data accuracy

219 and AOI size on classification performance of simulated fixations (on the forehead). (C)

220 Visualization of AOIs with $1.0^{\circ}$ and $2.0^{\circ}$ radius and the simulated fixation points on the forehead in

221 red $\left(n=100\right.$, averaged over 30 simulated fixations; accuracy $\left.0.5^{\circ}\right)$. (D) Green indicates the correct

222 classification outside any AOI, orange and gray indicate misclassifications as fixations within the

223 AOIs of the eye region or within the other AOIs.

$224 \quad 3.3 \quad$ Classification of fixations on the face 
226 by an ellipse drawn around the entire face with no specific facial features discriminated. To

227 investigate the effect of data accuracy on false-negatives, data was simulated on four facial targets

228 points (left eye, right eye, nose \& mouth) and classified as being directed towards or away from the

229 face. Accuracy values up to a $1^{\circ}$ degree visual angle allow nearly error-free classification of fixation

230 points $\left(0.5^{\circ}\right.$ accuracy: approx. $99 \%$ correct classification; $1.0^{\circ}$ accuracy: approx. $97 \%$ correct

231 classification), but even accuracy values of a $1.5^{\circ}$ degree visual angle reduce the classification

232 performance by only about 10\% (approx. 89\% correct classification) (see Fig 4B).

a)

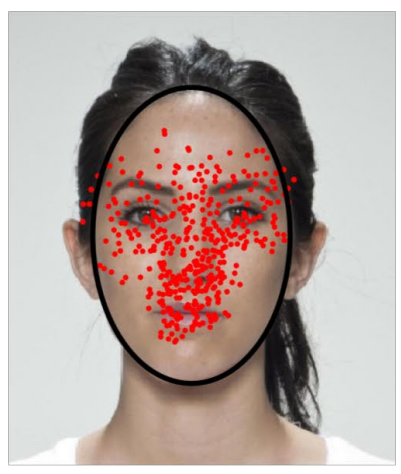

b)

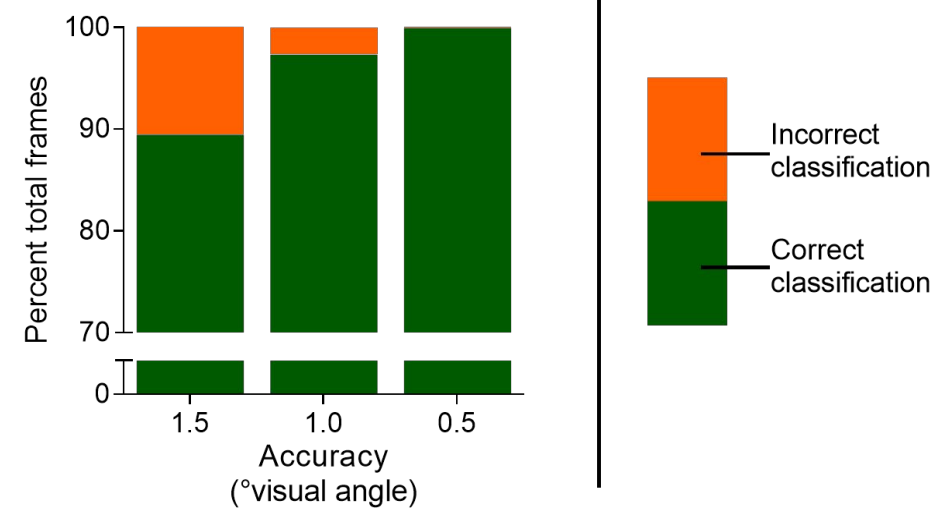

Fig 4. Effect of gaze data accuracy on classification performance of simulated fixations (on eyes, nose \& mouth) within the face ellipse.

(A) Visualization of the face ellipse and the simulated fixation points with an accuracy of $1.5^{\circ}(n=$ 100, averaged over 30 simulated fixations) in red. (B) Green indicates correct classification within

238 the face ellipse compared to orange for misclassification outside the face ellipse. 
FACIAL AREAS OF INTEREST IN EYE TRACKING

In the present simulation study, we investigated the effect of data accuracy and AOI size on gaze data classification for facial stimuli in a (simulated) interactive eye tracking setup. As on gaze data accuracy. Differentiating this effect in terms of Type I and Type II errors, we found that AOI size is irrelevant concerning Type II error (falsely classified outside predefined AOIs) for data accuracy better than $1.0^{\circ}$. The picture changed when data accuracy exceeded the threshold of $1.0^{\circ}$ within the present setup: larger AOIs increased classification accuracy leading to lower Type II errors. On the other hand, if we consider the Type I error (falsely classified inside a predefined AOI), the definition of smaller AOIs seems appropriate for all levels of data accuracy simulated here. However, the advantages of smaller AOIs decline with a data accuracy worse than $1.0^{\circ}$. Here the probability of misclassification or Type I errors increases.

Our findings regarding Type II error are consistent with previous accounts of the interaction between data accuracy and AOI size on gaze data classification performance $(20,21)$. Moreover, a systematic observation of gaze data classification performance on facial stimuli using the same AOI definition as in the present study concluded that larger AOIs are a noise-robust solution (13). Therefore, larger AOIs might be a better choice with data accuracy worse than $1.0^{\circ}$, but larger AOIs are also associated with higher Type I error when data accuracy is low. phobia or autism with such interactive eye tracking setups, for which small spatial differences in attention allocation are characteristic $(22,23)$. We simulated such a scenario by including fixations

262 directed at a target point on the forehead of the facial stimuli for which no AOI had been defined. We 263 chose this scenario because people suffering from social interaction disorders often report employing 


\section{FACIAL AREAS OF INTEREST IN EYE TRACKING}

264

265

266

267

268

269

270

271

272

273

274

275

276

277

278

279

280

281

282

283

284

285

strategies to normalize their gaze behavior in social interactions, such as looking between the eyes or at their interaction partner's forehead. Our simulation results are in line with another publication that recommended smaller AOIs to prevent inflated Type I errors (14). However, by examining a wide range of data accuracy in the current study, we additionally observed that the superiority of smaller AOIs decreases in conjunction with reduced data accuracy.

Given the differential effects on Type I and Type II errors, medium-sized AOIs would represent a compromise, but when we consider the data accuracy for values worse than $1.0^{\circ}$, only about $40 \%$ of fixations were correctly classified in both simulations. Therefore, we included a simulation designed for scenarios with low data accuracy that sacrifices facial feature distinction in favor of classification performance. While $1.5^{\circ}$ data accuracy resulted in a classification performance below the chance level when differentiating facial features, the face ellipse as the largest meaningful AOI in face perception resulted in almost $90 \%$ of the fixations being classified correctly even with data accuracy as low as $1.5^{\circ}$.

Based on the present simulation study, the recommendations for the choice of facial AOI size in interactive eye tracking setups can be summarized as follows:

- Inflation of Type I error (falsely classified gaze points inside an AOI) can be prevented by using small AOIs, such as radii of $1.0^{\circ}$, regardless of the data accuracy

- As previously published and recommended, the use of larger AOIs prevents the inflation of the Type II error (falsely classified gaze points outside an AOI), especially for data accuracy above $1.0^{\circ}$

- If both error types (Type I \& Type II errors) are to be compensated for in a setup with data accuracy of $1.0^{\circ}$ or better, smaller AOIs appear to be the better choice 


\section{FACIAL AREAS OF INTEREST IN EYE TRACKING}

- If both error types (Type I \& Type II errors) are to be compensated for in a setup with data accuracy worse than $1.0^{\circ}$, we suggest not using AOIs to distinguish facial features, but instead a face ellipse as an indicator of facial gaze

While the AOI size can be freely chosen by the investigator, the limiting factor is the data accuracy. Hence, it seems crucial not only to maximize the data accuracy as far as possible, but furthermore to estimate data accuracy based on the data set under the specific recording conditions. Ideally, distinct trials instructing the participants to fixate predefined points in the tracking area are included in the study protocol that allow the proper calculation of data accuracy. If this is not possible, attempts should be made to estimate the data accuracy from data recorded under the specific conditions, which are often imperfect and often not comparable to the conditions on which the manufacturers base their specifications.

One potential limitation might be that the present simulation was based on the assumption that gaze data follows a unique distribution for all generated data sets. Although, the overall parameters (deviation and skewness) are largely in accordance with data recorded in the real setup underlying the present simulation (12), it is possible that other recording conditions, populations or interaction paradigms will lead to different data distributions. Therefore, we make the gaze data simulation tool used in the present study freely available and encourage repeating or modifying the simulation process.

Furthermore, one might question the use of AOIs in general due to their subjective nature and the fact that the definition can be very time-consuming especially with moving AOIs in interactive setups (24). We tried to minimize these practical disadvantages by using a validated automatic AOIdefinition method that takes a Voronoi-Tessellation approach to avoid subjectivity, and the OpenFace tool to save time. Nevertheless, data-driven approaches based on neural networks $(25,26)$ could be an 


\section{FACIAL AREAS OF INTEREST IN EYE TRACKING}

309 alternative approach when data quality is uncertain or difficult to estimate, an approach that could be

310 evaluated in future studies when used in interactive eye tracking setups.

311 It should be noted that visual angle values used in the present study need to be adjusted to

312 differing viewing distances for meaningful comparison between studies. Thus, lower data accuracy

313 can be in part compensated by using shorter viewing distances, as long as the setup allows shorter

314 viewing distances. In turn, longer viewing distances or situations with stimuli which are observed at

315 varying viewing distances should be treated with caution. However, future studies can apply the

316 general recommendations proposed in the present paper to justify the choice of AOI size depending

317 on data accuracy and viewing distance in the given setup and environment.

318 The present study thus might help to further improve reporting standards in eye tracking

319 research $(20,21,27)$. Furthermore, the freely available simulation tool used in the present study can be

320 used to support the validation process of novel eye tracking setups.

321 Eye tracking during real social interactions is a powerful tool to examine social attention and

322 behavior in both healthy and clinical populations. Data accuracy in these setups can be compromised

323 by movements caused by speech, facial expressions, or head rotations. It is thus essential to validate

324 novel interactive eye tracking setups carefully. The proper estimation of data accuracy is an essential

325 prerequisite for informed decisions regarding the size of AOIs used for data analysis. The results of

326 the present simulation study indicate that smaller AOIs minimize false classifications (both Type I

327 and Type II errors) as long as data accuracy is good enough. For studies with lower data accuracy,

328 Type II errors can still be compensated to some extent by using larger AOIs, but at the cost of

329 increasing the probability of Type I errors. When data accuracy is low, facial feature discrimination

330 should better be omitted and larger AOIs, such as a face ellipse, should be preferred to allow valid

331 AOI classification. 
333 1. Kaiser D, Jacob GA, van Zutphen L, Siep N, Sprenger A, Tuschen-Caffier B, et al. Biased 334 attention to facial expressions of ambiguous emotions in borderline personality sisorder: An eye-tracking study. J Pers Disord. 2019 Jan 28;33(5):1-28.

2. Mojzisch A, Schilbach L, Helmert JR, Pannasch S, Velichkovsky BM, Vogeley K. The effects

5. Reilly JL, Lencer R, Bishop JR, Keedy S, Sweeney JA. Pharmacological treatment effects on

6. Rubin M, Minns S, Muller K, Tong MH, Hayhoe MM, Telch MJ. Avoidance of social threat: of self-involvement on attention, arousal, and facial expression during social interaction with virtual others: A psychophysiological study. Soc Neurosci. 2006 Sep;1(3-4):184-95.

3. Domes G, Steiner A, Porges SW, Heinrichs M. Oxytocin differentially modulates eye gaze to naturalistic social signals of happiness and anger. Psychoneuroendocrinology. 2013 Jul;38(7):1198-202.

4. Lischke A, Berger C, Prehn K, Heinrichs M, Herpertz SC, Domes G. Intranasal oxytocin enhances emotion recognition from dynamic facial expressions and leaves eye-gaze unaffected. Psychoneuroendocrinology. 2012 Apr;37(4):475-81.

eye movement control. Brain Cogn. 2008 Dec;68(3):415-35.

Evidence from eye movements during a public speaking challenge using $360^{\circ}$ - video. Behav Res Ther. 2020 Nov;134:Article 103706.

7. Vatheuer CC, Vehlen A, Dawans B von, Domes G. Gaze behavior is associated with the cortisol response to acute psychosocial stress in the virtual TSST. J Neural Transm [Internet]. 2021 [cited 2021 Apr 30]; Available from: https://link.springer.com/epdf/10.1007/s00702-021-02344$\mathrm{W}$

8. Dalrymple KA, Manner MD, Harmelink KA, Teska EP, Elison JT. An examination of recording accuracy and precision from eye tracking data from toddlerhood to adulthood. Front Psychol. 2018;9:1-12.

9. Niehorster DC, Santini T, Hessels RS, Hooge ITC, Kasneci E, Nyström M. The impact of slippage on the data quality of head-worn eye trackers. Behav Res Methods. 2020 Jun 1;52(3):1140-60.

10. Hessels RS, Cornelissen THW, Hooge ITC, Kemner C. Gaze behavior to faces during dyadic interaction. Can J Exp Psychol. 2017 Sep;71(3):226-42.

11. Grossman RB, Zane E, Mertens J, Mitchell T. Facetime vs. screentime: Gaze patterns to live and video social stimuli in adolescents with ASD. Sci Rep. 2019 Sep 2;9(1):1-10.

12. Vehlen A, Spenthof I, Tönsing D, Heinrichs M, Domes G. Evaluation of an eye tracking setup 


\section{FACIAL AREAS OF INTEREST IN EYE TRACKING}

13. Hessels RS, Kemner C, van den Boomen C, Hooge ITC. The area-of-interest problem in eyetracking research: A noise-robust solution for face and sparse stimuli. Behavior Research Methods. 2016 Dec;48(4):1694-712.

14. Orquin JL, Ashby NJS, Clarke ADF. Areas of interest as a signal detection problem in behavioral eye-tracking research: Areas of interest as a signal detection problem. J Behav Dec Making. 2016 Apr 7;29(2-3):103-15.

15. Cañigueral R, Hamilton $F$ de CA. The role of eye gaze during natural social interactions in typical and autistic people. Front Psychol [Internet]. 2019 Mar 15 [cited 2019 Dec 24];10. Available from: https://www.ncbi.nlm.nih.gov/pmc/articles/PMC6428744/

16. Hessels RS, Holleman GA, Cornelissen THW, Hooge ITC, Kemner C. Eye contact takes two Autistic and social anxiety traits predict gaze behavior in dyadic interaction. J Exp Psychopathol. 2018;1-17.

17. Hessels RS, Benjamins JS, Cornelissen THW, Hooge ITC. A validation of automaticallygenerated areas-of-interest in videos of a face for eye-tracking research. Front Psychol. 2018 Aug 3;9:Article 1367.

18. DeBruine L, Jones B. Face Research Lab London Set. [Internet]. 2017. Available from: https://figshare.com/arti cles/Face_Research_Lab_London_Set/5047666

19. Amos B, Ludwiczuk B, Satyanarayanan M. OpenFace: A general-purpose face recognition library with mobile applications. CMU-CS-16-118, CMU School of Computer Science; 2016 p. $1-18$.

20. Holmqvist K, Nyström M, Mulvey F. Eye tracker data quality: What it is and how to measure it. In: Proceedings of the Symposium on Eye Tracking Research and Application [Internet]. Santa Barbara, California: ACM Press; 2012 [cited 2020 Jan 7]. p. 45-52. Available from: http://dl.acm.org/citation.cfm?doid=2168556.2168563

21. Orquin JL, Holmqvist K. Threats to the validity of eye-movement research in psychology. Behav Res Methods. 2018 Aug 1;50(4):1645-56.

22. Chita-Tegmark M. Attention allocation in ASD: A review and meta-analysis of eye-tracking studies. J Autism Dev Disord. 2016 Sep 1;3(3):209-23.

23. Chen NTM, Clarke PJF. Gaze-based assessments of vigilance and avoidance in social anxiety: A review. Curr Psychiatry Rep. 2017 Jul 20;19(9):Article 59.

24. Rim NW, Choe KW, Scrivner C, Berman MG. Introducing point-of-interest as an alternative to area-of-interest for fixation duration analysis. PLOS ONE. 2021 May 10;16(5):Article $\mathrm{e} 0250170$.

25. Fuhl W, Bozkir E, Hosp B, Castner N, Geisler D, Santini TC, et al. Encodji: Encoding gaze data into emoji space for an amusing scanpath classification approach;). In: Proceedings of the 11th ACM Symposium on Eye Tracking Research \& Applications [Internet]. Denver, Colorado: ACM Press; 2019 [cited 2021 May 27]. p. 1-4. Available from: https://dl.acm.org/doi/10.1145/3314111.3323074 
405 26. Castner N, Kuebler TC, Scheiter K, Richter J, Eder T, Huettig F, et al. Deep semantic gaze

406

407

408

409 embedding and scanpath comparison for expertise classification during OPT viewing. In: ACM Symposium on Eye Tracking Research and Applications [Internet]. Stuttgart Germany: ACM Press; 2020 [cited 2021 May 25]. p. 1-10. Available from: https://dl.acm.org/doi/10.1145/3379155.3391320

410 27. Carter BT, Luke SG. Best practices in eye tracking research. Int J Psychophysiol. 2020

411 Sep;155:49-62.

412

413

414

415

416

417

418

419

420

421

422

423

424

425 
4266 Competing interest statement

427 The authors declare that the research was conducted in the absence of any commercial or

428 financial relationships that could be construed as a potential conflict of interest.

429

\section{$430 \quad 7 \quad$ Funding}

431 The study was in part supported by grants from the German Research Foundation (DO1312/5-

432 1) and Trier University Research Priority Program "Psychobiology of Stress", funded by the State

433 Rhineland-Palatinate.

434

4358 Data Availability Statement

436 The datasets generated and analyzed in this study can be reproduced using the descripted

437 software tool, which can be downloaded from the following website:

438 https://osf.io/ytrh8/?view_only=bedda3995dba447bb58f81c2f8c27880

439 